\title{
Review: little evidence supports the efficacy of major commercial and organised self help weight loss programmes
}

Tsai AG, Wadden TA. Systematic review: an evaluation of major commercial weight loss programs in the United States. Ann Intern Med 2005; 142:56-66.

What is the efficacy of major commercial or organised self help weight loss programmes that provide structured inperson or online counselling?

\section{METHODS}

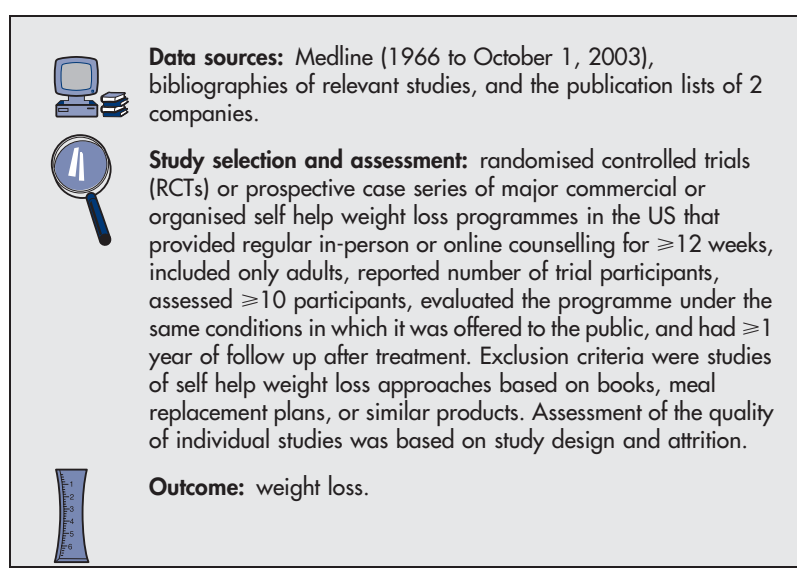

\section{MAIN RESULTS}

10 studies $(\mathrm{n}=1879)$ met the selection criteria. 3 RCTs $(\mathrm{n}=551)$ assessed a non-medical programme (Weight Watchers). Of these, 1 RCT $(n=423)$ reported that participants attending Weight Watchers meetings had a mean loss of $3.2 \%$ of initial weight at 2 years compared with no participants assigned to a self help intervention that included 2 dietitian visits $(\mathrm{p}<0.001)$

5 studies $(\mathrm{n}=1048)$ assessed physician supervised weight loss programmes, 3 of Health Management Resources (Boston, Massachusetts) and 2 of OPTIFAST (Novartis Nutrition, Minneapolis, Minnesota). Both programmes offered meal replacement products as part of a low calorie diet or very low calorie diet (VLCD) with a curriculum of lifestyle modification. 1 of these 5 studies was an RCT. The RCT $(\mathrm{n}=40)$ compared meal replacement products alone with meal replacement products plus usual table foods in participants with type 2 diabetes and obesity but did not report between-group differences in weight loss at 1 year.

1 RCT $(\mathrm{n}=46)$ assessed a commercial, internet based, weight loss programme (eDiets.com). At 1 year, participants in the internet based programme lost $1.1 \%$ of their initial weight compared with $4.0 \%$ in participants who used a behavioural weight loss manual $(\mathrm{p}=0.04)$.

I RCT $(n=234)$ assessed an organised self help programme (Take Off Pounds Sensibly [TOPS]). At 1 year, the TOPS programme was less effective than a therapist led behavioural modification programme for achieving weight loss ( $\mathrm{p}$ value for difference between groups not provided).

For correspondence: Dr T A Wadden, University of Pennsylvania, Philadelphia, Pennsylvania, USA. wadden@mail.med.upenn.edu

Source of funding: National Institutes of Health.

\section{CONCLUSIONS}

1 of 5 randomised controlled trials showed that a non-medical commercial weight loss programme (Weight Watchers) was modestly effective in achieving long term weight loss. Little evidence supports the efficacy of commercial and self help weight loss programmes.

A modified version of this abstracts appears in ACP Journal Club.

Commentary

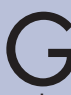
iven the serious health implications of obesity, clinicians need to have scientifically accurate information about weight loss programmes in order to counsel clients. In the review by Tsai and Wadden, 10 studies were selected from $>1500$ identified abstracts. Only US studies were evaluated despite the availability of programmes in other countries and of VLCD meal replacements via the internet. Statistical analysis was not done because of data limitations of the included studies. The review also included 1 study of VLCDs, which reported that VLCDs were effective for weight loss; this contradicts previous conclusions that VLCDs yield similar results to low calorie diets at 1 year. ${ }^{12}$ Because participants were adults with obesity, findings may not be relevant for adults who are overweight (body mass index $27-30 \mathrm{~kg} / \mathrm{m}^{2}$ ) or for other groups (eg, different ethnic groups) and thus warrant cautious interpretation.

The review findings accentuate the fact that we do not have a best treatment for sustaining weight loss and that further research is needed to assess long term outcomes. Nevertheless, the results are useful to nurse clinicians in public health, family, women's health, and primary care settings. Before suggesting a weight loss programme, nurses could explore weight loss history and expectations, and discuss costs, staff qualifications, and programme components. A person needing much emotional support could consider programmes with individual counselling. Persons considering VLCDs could be advised that ongoing medical monitoring is needed while on these diets and that research findings on their effectiveness are limited to short term durations (12-26 wks). Because high attrition rates are reported, nurses can help plan weight loss strategies that sustain motivation during long term participation. Kathryn Weaver, RN, MN EQUIPP Pre-doctoral Fellow Canadian Institutes of Health Research and International Institute for Qualitative Methodology University of Alberta Edmonton, Alberta, Canada

1 US Department of Health and Human Services. Clinical guidelines on the identification, evaluation, and treatment of overweight and obesity in adults: the evidence report. NIH Publication No 98-4083, September 1998. http://www.nhlbi.nih.gov/guidelines/obesity/ob_gdlns.pdf

2 Wadden TA, Foster GD, Letizia KA. One-year behavioral treatment of obesity: comparison of moderate and severe caloric restriction and the effects of weight maintenance therapy. J Consult Clin Psychol 1994;62:165-71. 